\title{
Towards quantitative viromics for both double-stranded and single-stranded DNA viruses
}

\author{
Simon Roux ${ }^{1}$, Natalie E Solonenko ${ }^{1}$, Vinh T Dang ${ }^{2}$, Bonnie T Poulos ${ }^{3}$, Sarah M Schwenck ${ }^{3}$, Dawn B

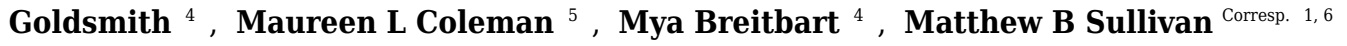 \\ 1 Department of Microbiology, The Ohio State University, Columbus, Ohio, United States \\ 2 Department of Microbiology, Ha Long University, Uong Bi, Quang Ninh, Vietnam \\ 3 Department of Ecology and Evolutionary Biology, University of Arizona, Tucson, Arizona, United States \\ 4 College of Marine Science, University of South Florida, St. Petersburg, Florida, United States \\ 5 Department of the Geophysical Sciences, University of Chicago, Chicago, Illinois, United States \\ 6 Department of Civil, Environmental and Geodetic Engineering, The Ohio State University, Columbus, Ohio, United States \\ Corresponding Author: Matthew B Sullivan \\ Email address: mbsulli@gmail.com
}

Background. Viruses strongly influence microbial population dynamics and ecosystem functions. However, our ability to quantitatively evaluate those viral impacts is limited to the few cultivated viruses and double-stranded DNA (dsDNA) viral genomes captured in quantitative viral metagenomes (viromes). This leaves the ecology of non-dsDNA viruses nearly unknown, including single-stranded DNA (ssDNA) viruses that have been frequently observed in viromes, but not quantified due to amplification biases in sequencing library preparations (Multiple Displacement Amplification, Linker Amplification or Tagmentation). Methods. Here we designed mock viral communities including both ssDNA and dsDNA viruses to evaluate the capability of a sequencing library preparation approach including an Adaptase step prior to Linker Amplification for quantitative amplification of both dsDNA and ssDNA templates. We then surveyed aquatic samples to provide first estimates of the abundance of ssDNA viruses. Results. Mock community experiments confirmed the biased nature of existing library preparation methods for ssDNA templates (either largely enriched or selected against) and showed that the protocol using Adaptase plus Linker Amplification yielded viromes that were +/- 1.8-fold quantitative for ssDNA and dsDNA viruses. Application of this protocol to community virus DNA from 3 freshwater and 3 marine samples revealed that ssDNA viruses as a whole represent only a minor fraction $(<5 \%)$ of DNA virus communities, though individual SsDNA genomes, both eukaryoteinfecting Circular Rep-Encoding Single-Stranded DNA (CRESS-DNA) viruses and bacteriophages from the Microviridae family, can be among the most abundant viral genomes in a sample. Discussion. Together these findings provide empirical data for a new virome library preparation protocol, and a first estimate of ssDNA virus abundance in aquatic systems. 


\title{
Towards quantitative viromics for both double-stranded and single-stranded DNA viruses
}

\author{
Simon Roux ${ }^{1}$, Natalie Solonenko ${ }^{1}$, Vinh T Dang ${ }^{2}$, Bonnie T Poulos ${ }^{3}$, Sarah M Schwenck ${ }^{3}$, Dawn
} B Goldsmith ${ }^{4}$, Maureen L Coleman ${ }^{5}$, Mya Breitbart ${ }^{4}$, Matthew B Sullivan ${ }^{1,6^{*}}$

${ }^{1}$ Department of Microbiology, The Ohio State University, Columbus, OH, USA

$5 \quad{ }^{2}$ Department of Microbiology, Ha Long University, Uong Bi, Quang Ninh, Vietnam

${ }^{3}$ Department of Ecology and Evolutionary Biology, University of Arizona, Tucson, AZ, USA

${ }^{4}$ College of Marine Science, University of South Florida, St. Petersburg, FL, USA

${ }^{5}$ Department of the Geophysical Sciences, University of Chicago, Chicago, IL, USA

${ }^{6}$ Department of Civil, Environmental and Geodetic Engineering, The Ohio State University,

10 Columbus, $\mathrm{OH}$, USA

*correspondence to mbsulli@gmail.com

\begin{abstract}
Background. Viruses strongly influence microbial population dynamics and ecosystem functions. However, our ability to quantitatively evaluate those viral impacts is limited to the few

15 cultivated viruses and double-stranded DNA (dsDNA) viral genomes captured in quantitative viral metagenomes (viromes). This leaves the ecology of non-dsDNA viruses nearly unknown, including single-stranded DNA (ssDNA) viruses that have been frequently observed in viromes, but not quantified due to amplification biases in sequencing library preparations (Multiple Displacement Amplification, Linker Amplification or Tagmentation).

20 Methods. Here we designed mock viral communities including both ssDNA and dsDNA viruses to evaluate the capability of a sequencing library preparation approach including an Adaptase step prior to Linker Amplification for quantitative amplification of both dsDNA and ssDNA templates. We then surveyed aquatic samples to provide first estimates of the abundance of ssDNA viruses.

25 Results. Mock community experiments confirmed the biased nature of existing library preparation methods for ssDNA templates (either largely enriched or selected against) and showed that the protocol using Adaptase plus Linker Amplification yielded viromes that were +/1.8-fold quantitative for ssDNA and dsDNA viruses. Application of this protocol to community virus DNA from 3 freshwater and 3 marine samples revealed that ssDNA viruses as a whole 30 represent only a minor fraction $(<5 \%)$ of DNA virus communities, though individual ssDNA genomes, both eukaryote-infecting Circular Rep-Encoding Single-Stranded DNA (CRESS-DNA) viruses and bacteriophages from the Microviridae family, can be among the most abundant viral genomes in a sample.
\end{abstract}

Discussion. Together these findings provide empirical data for a new virome library preparation protocol, and a first estimate of ssDNA virus abundance in aquatic systems. 


\section{Main text}

Introduction

It is now increasingly clear that microorganisms play a central role in all of Earth's ecosystems and processes. In every biome - from the human gut to the oceans, soils, and extreme 40 environments that challenge life to succeed - microbes drive the nutrient and energy transformations that fuel these ecosystems (Falkowski, Fenchel \& Delong, 2008; Sommer \& Bäckhed, 2013). Microbial diversity was first revealed through universal marker genes studies (Pace, 1997), and has now advanced to genome-level characterizations helping revise our understanding of the microbial tree of life (Rinke et al., 2013; Hug et al., 2016), as well as

45 elucidate the ecological and evolutionary roles of lesser-studied microbial lineages (Wrighton et al., 2012; Castelle et al., 2013; Brown et al., 2015).

Recent technical and theoretical advances are now also revealing that these microbial roles are modulated by co-occurring and co-evolving viruses (Weitz et al., 2015; O’Malley, 2016). Viruses are the most abundant biological entities on Earth, and have profound impacts on their microbial 50 hosts through mortality, horizontal gene transfer and metabolic reprogramming (Fuhrman, 1999; Suttle, 2007; Rohwer \& Thurber, 2009). Since most microbes and viruses remain uncultivated and viruses do not harbor a universal marker gene, community-level surveys of viruses typically rely on laboratory culture or reference-independent methods such as viral metagenomics (a.k.a. viromics). These studies have provided a view of viral diversity that complements knowledge

55 obtained from isolate collections, and revealed new viral groups, evolutionary patterns, and virus-host interactions in multiple systems (Edwards \& Rohwer, 2005; Mokili, Rohwer \& Dutilh, 2012; Fancello, Raoult \& Desnues, 2012; Brum \& Sullivan, 2015).

Because natural community samples typically yield limiting input DNA, multiple displacement amplification (MDA) or whole genome amplification (WGA) are commonly used prior to

60 sequencing library preparation in viromics studies (Edwards \& Rohwer, 2005; Brum \& Sullivan, 2015). While these MDA viromes routinely uncover new viruses (Angly et al., 2006, 2009; Wegley et al., 2007; Kim et al., 2008; Willner et al., 2009; Ng et al., 2009; Rosario, Duffy \& Breitbart, 2009; Rosario et al., 2009; López-Bueno et al., 2009; Roux et al., 2012a,b; Labonté \& Suttle, 2013a,b; Zawar-Reza et al., 2014; Quaiser et al., 2015; Dayaram et al., 2016; Male et al., 65 2016; Steel et al., 2016), the MDA step selects for small circular ssDNA templates, and unevenly amplifies linear genome fragments even when pooling independent reactions (Yilmaz, Allgaier \& Hugenholtz, 2010; Kim \& Bae, 2011; Marine et al., 2014). The alternative linker amplification (LA) or tagmentation (TAG) methods are quantitative $(+/-1.5$-fold) for dsDNA viruses, even from low input samples (100 femtograms, (Duhaime et al., 2012)) but strongly select against 70 ssDNA templates (Kim \& Bae, 2011). This leaves researchers to choose between quantitatively studying dsDNA viruses alone or pursuing questions constrained to discovery rather than ecology if interested in both ssDNA and dsDNA viruses.

The recently available Swift Biosciences 1S Plus kit for preparing sequencing libraries incorporates an adaptase step prior to linker ligation and amplification, which makes it efficient

75 for both ssDNA and dsDNA templates (Kurihara et al., 2014; Aigrain, Gu \& Quail, 2016). Here we use replicated metagenomic experiments to evaluate this protocol, hereafter named A-LA for Adaptase-Linker Amplification, alongside two existing protocols (MDA and TAG) for their ability to quantitatively amplify ssDNA and dsDNA viruses from two mock viral communities. Then, we apply the methods to aquatic samples known to harbor ssDNA viruses and estimate the relative abundance of ssDNA viruses. 
$\underline{\text { Material \& Methods }}$

Mock community generation

The ten dsDNA phages included in the mock communities were grown on Pseudoalteromonas or Cellulophaga baltica (Table 1, (Duhaime et al., 2011; Holmfeldt et al., 2013)). These were 85 selected to represent the three main families of dsDNA bacteriophages (Myoviridae, Podoviridae, and Siphoviridae), a range of genome length (35-130kb) and GC \% (30-45\%). The two ssDNA phages included were phiX174 and alpha 3, representing two distinct clades in the well-characterized Microvirus genus (from the Microviridae family), both grown on Escherichia coli (Rokyta et al., 2006).

90 Two mock communities were designed (A and B) corresponding to two contrasting situations with either low abundance of ssDNA viruses (MCA, total ssDNA $\sim 2 \%$ of community) or high abundance of ssDNA viruses (MCB, total ssDNA $\sim 66 \%$ of community).

Each virus to be included in the mock community was grown on its specific host, and viral capsids were obtained from lysates. The concentration of viral capsids was determined through

95 SYBR Gold counting (Noble, 2001), using the wet-mound method (Cunningham et al., 2015), and two mixes of viral capsids corresponding to the desired relative proportion of viruses were created (MCA and MCB, Table 1). Although epifluorescence enumeration of some ssDNA phages can be challenging (Holmfeldt et al., 2012), SYBR-stained micrographs from phiX174 and alpha 3 were readily countable (Fig. S1).

100 For each mix, DNA was extracted with the QIAamp DNA Mini Kit (Qiagen 51304). Triplicate viromes were generated using DNA extracted from these two mock communities with three different sequencing library protocols (A-LA, TAG and MDA). This experimental design allowed us to evaluate the potential influence of both the DNA extraction step (same bias across all methods, as the same pool of DNA was used as input for all methods in MCA and MCB

105 samples) and the DNA amplification step (different biases between methods) at three different levels: (i) the relative proportion of ssDNA vs dsDNA viruses, (ii) the relative proportion of individual genomes within ssDNA and dsDNA virus communities, and (iii) the coverage variation within a genome. The MDA library was generated using the GE HealthCare GenomiPhi v2 DNA Amplification Kit followed by NexteraXT DNA Library Preparation Kit, the TAG

110 library using the standard NexteraXT DNA Library Preparation Kit, and the A-LA library with the Swift 1S Plus DNA Library Kit for Illumina. All samples were sequenced on the Illumina MiSeq platform.

\section{Mock community viromes: read quality control, assembly, and annotation}

Raw reads were curated with Trimmomatic to remove adaptors, trim reads as soon as the base-

115 calling quality dropped below 20 on a $4 \mathrm{bp}$ sliding window, and remove reads shorter than $50 \mathrm{bp}$ (Bolger, Lohse \& Usadel, 2014). Trimmed and filtered reads from mock communities were mapped to the 12 reference genomes with Bowtie 2 (--non-deterministic option, default options otherwise, (Langmead \& Salzberg, 2012)), and the normalized coverage of each genome (i.e. number of base pairs mapped at $100 \%$ identity to the genome normalized by the genome length

120 and total number of base pairs sequenced in the virome) was used as a proxy for the relative abundance of each viral genome. Using a normalized coverage (i.e. number of reads mapped per position) rather than the total number of mapped reads per genome for estimating the relative abundance of each virus means that these relative abundance values did not have to be corrected for the different genome sizes. The expected number of viral genomes was calculated from the 
125 number of viral particles from each virus added in each mix, taking into account the fact that dsDNA viruses would provide twice as many genome copies as ssDNA viruses per particle following the first denaturation step of library preparation, and accounting for the low extraction efficiency of dsDNA genomes from lysates with the QIAamp DNA Mini Kit (estimated at 27\% of DNA successfully recovered for the dsDNA viral genomes in these mixes; no similar bias was

130 observed for ssDNA viral genomes). This DNA extraction efficiency was calculated based on the ratio between expected total DNA concentration (based on SYBR counts and the known genome size of the virus) and the observed DNA concentration (measured with PicoGreen) for PSA-HM1 (Table S1). Hence, the expected relative abundance of viruses in MCA and MCB viromes (Table 1) are calculated based on the expected number of genomes in the mix normalized by this

135 dsDNA extraction bias. To verify if complete and accurate genomes could be reconstructed de novo from the mock community virome reads, the QC'd reads were assembled with Spades 3.6.2 using options "sc" and "careful", default options otherwise (Bankevich et al., 2012), and contigs $\geq 500 \mathrm{bp}$ were compared to reference genomes with Nucmer (Delcher, Salzberg \& Phillippy, 2003).

140 Within dsDNA viruses, the influence of mock community (A or B), library preparation method (MDA, TAG, A-LA), and genome on relative abundance (which should theoretically be $10 \%$ for every genome) was investigated with Kruskal-Wallis tests. Each genome's relative abundance was compared between A-LA and TAG libraries using a Wilcoxon two-sided test (Fig. S2). Similarly, statistically different ranges of coverage variations for each genome between the

145 different library preparation methods were assessed with Wilcoxon two-sided tests (Fig. S3). All plots and tests were conducted with the R software (R Core Team, 2016) and the ggplot2 module (Wickham, 2009).

\section{Environmental virome sampling and processing}

For freshwater lakes, three integrated water column samples were taken in April 2013 in Lake 150 Superior (SU08M), Lake Erie (ER15M), and Lake Michigan (MI41M). Samples from three different depths (a "surface" sample at $\sim 2 \mathrm{~m}$ for all lakes, an "intermediate" sample at $30 \mathrm{~m}$ for Lake Erie, $100 \mathrm{~m}$ for Lake Michigan and Superior, and a "deep" sample at $53 \mathrm{~m}$ for Lake Erie, 249m for Lake Michigan, and 282m for Lake Superior) were combined, since the lakes were not stratified at the time of the sampling. For each lake, 33 to $45 \mathrm{~L}$ of water were $0.22 \mu \mathrm{m}$-filtered and

155 viruses were concentrated from the filtrate using iron chloride flocculation (John et al., 2011) followed by storage at $4^{\circ} \mathrm{C}$. One seawater sample originates from the Tara Oceans expedition collection (sample T102S) and was processed as previously described (Pesant et al., 2015). Briefly, $20 \mathrm{~L}$ of seawater were $0.22 \mu \mathrm{m}$-filtered, and viruses were concentrated from the filtrate using iron chloride flocculation (John et al., 2011) followed by storage at $4^{\circ} \mathrm{C}$. In both cases, after 160 resuspension in ascorbic-EDTA buffer (0.1 M EDTA, 0.2 M Mg, 0.2 M ascorbic acid, pH 6.0), viral particles were concentrated using Amicon Ultra $100 \mathrm{kDa}$ centrifugal devices (Millipore), treated with DNase I $(100 \mathrm{U} / \mathrm{mL})$ followed by the addition of $0.1 \mathrm{M}$ EDTA and $0.1 \mathrm{M}$ EGTA to halt enzyme activity, and extracted with the QIAamp DNA Mini Kit (Qiagen 51304). The two remaining water samples were collected from $0 \mathrm{~m}$ and $100 \mathrm{~m}$ depths at the Bermuda Atlantic

165 Time-series Study site in March 2011, where approximately 180L of seawater were concentrated using a $100 \mathrm{kDa}$ tangential flow filter, $0.22 \mu \mathrm{m}$-filtered, PEG precipitated, cesium chloride purified, and DNA was extracted using formamide (Goldsmith et al., 2015). All samples were sequenced on an Illumina MiSeq platform at the University of Arizona Genetics Core. 
Environmental viromes read quality control, assembly, and identification of viral contigs

170 For freshwater and seawater samples, trimmed and filtered reads (generated as for the mock community datasets, see above) for all libraries (MDA, TAG, and A-LA) were pooled for each sample and assembled with Spades 3.6.2 with the "sc" and "careful" options (Bankevich et al., 2012). All contigs $>500 \mathrm{bp}$ and with at least one complete gene were retained (representing on average $75 \%$ of trimmed and filtered reads, Table S5), and mined for contaminating cellular

175 sequences. Contigs $\geq 5 \mathrm{~kb}$ were run through VirSorter (Roux et al., 2015) in the "virome decontamination" mode, all contigs not detected as viral were excluded from the final datasets, and prophage predictions were manually curated to distinguish cellular sequences from erroneous predictions (i.e. viral sequences wrongly identified as a prophage). Another pipeline was applied to identify smaller viral contigs $(<5 \mathrm{~kb})$, which can be missed by VirSorter according

180 to simulations (Roux et al., 2015): sequences with no significant BLAST hit (bit score $>50$ and evalue $<10^{-3}$ ) against RefSeqVirus (i.e. no viral gene) and one significant hit (score $>50$ ) against PFAM (i.e. one "known" gene) were considered as cellular and thus excluded (with the exception of "viral" PFAM domain, i.e. PFAM domains with the keyword "viral", "phage", "capsid", "virion", "terminase", "tail", or "portal"). This allowed us to keep in the dataset both

185 sequences similar to known viruses, and sequences entirely new (i.e. all uncharacterized genes), which in a virome are most likely viral.

\section{Annotation of viral contigs from environmental viromes}

QC'd reads from individual libraries were then mapped back to the contigs with Bowtie 2 (-non-deterministic option, default options otherwise, (Langmead \& Salzberg, 2012)) to evaluate

190 the relative abundance of each sequence with each preparation method: contigs were considered detected in a library when $\geq 50 \%$ of the contig was covered, and the contig relative abundance was calculated from the contig average coverage normalized by the number of bp sequenced in the library. Contig affiliation was based on best BLAST hit against RefSeqVirus (thresholds: bit score $>50$ and e-value $<10^{-3}$ ). Contigs with best BLAST hits to only ssDNA or dsDNA viruses

195 were considered ssDNA or dsDNA viruses respectively, while the genome type of contigs with no hits or mixed affiliations (i.e. hits to both ssDNA and dsDNA reference genomes) was predicted based on their coverage in the different libraries: contigs detected in TAG libraries were considered dsDNA, while contigs only detected in MDA and/or A-LA were predicted as ssDNA. To take into account the fact that dsDNA genomes will provide twice as many templates

200 than ssDNA genomes per single virus in A-LA and MDA viromes (because the first step of the protocol is dsDNA denaturation), the coverage of all affiliated and predicted dsDNA contigs was divided by 2 , so that the relative proportion of contigs are approaching the relative proportion of viral particles in the sample.

\section{Scripts and datasets availability}

205 All scripts and datasets used in this study are available on iVirus (CyVerse, http://mirrors.iplantcollaborative.org/browse/iplant/home/shared/iVirus/DNA Viromes_library_c omparison), as well as https://bitbucket.org/MAVERICLab/dna_viromes_library_comparison (for scripts).

\section{$\underline{\text { Results \& Discussion }}$}

210 Mock community benchmarking for ssDNA and dsDNA genomic amplification

Two mock communities containing a minority (MCA) or majority (MCB) of ssDNA viruses 
were established from 2 ssDNA and 10 dsDNA viruses (Supplementary Material \& Methods, Table 1). DNA was extracted from each mock community, used as source material for constructing replicate sequencing libraries using MDA, TAG and A-LA methods, and sequenced

215 to create viromes with approximately 1,000-fold coverage for abundant viruses (Table S2).

As qualitatively observed previously (Kim \& Bae, 2011), and here quantified, MDA systematically favored ssDNA viruses ( 30- to 40-fold), whereas TAG systematically selected against them ( $\sim 30$ - to 100-fold, Fig. 1A, Table S2). In contrast, A-LA correctly recovered the proportion of ssDNA viruses when they were in the majority (MCB, 1.1-fold variation), and

220 slightly underestimated ssDNA viruses when they were in the minority (MCA, 1.8-fold variation, Fig. 1A, Table S2). These observations for all treatments were repeatable across duplicate or triplicate viromes (Fig. 1A). For A-LA viromes, the relative abundance of individual ssDNA viruses (within the ssDNA pool) was consistent across replicates, although not across the two mock communities (Table S2). This consistency across replicates suggests that the quantitative amplification of ssDNA viral communities through A-LA viromes is reproducible.

We next examined the variation in relative abundance estimates for the ten individual dsDNA viruses within the dsDNA pool in the mock communities, which revealed two main findings. First, the relative abundances of dsDNA viruses within the mock community were more variable than expected: each individual virus should represent $10 \%$ of the total dsDNA virus coverage,

230 while observed relative abundances ranged from 0 to $30 \%$ (Fig. S2). Specifically, genome, and not sample or method, was the only significant factor explaining differences in relative abundance in a multi-factorial analysis (Kruskal-Wallis test, p-value <2.2e-16, Fig. S2). The variation in relative abundances of each genome could be due to inaccurate viral particle counts and/or variable DNA extraction efficiencies for the input viruses. Notably, however, these

235 relative abundance deviations are minimal $(10 \% \pm 7-10$, average \pm st. dev. $)$ compared to the many-fold variation typically tolerable in viral ecological counts (Cunningham et al., 2015). Moreover, these per-genome relative abundance estimates were minimally impacted by the choice of library preparation method: for each individual genome, the relative abundances were not significantly different (Wilcoxon test, $p$-value $>0.01$, effect $s i z e<0.8$ ) for 6 of the 10 genomes

240 between TAG and A-LA (Fig. S2, Table S2). This suggests that the current method used for dsDNA viruses (TAG) and the method evaluated here (A-LA) provide a relatively similar view of dsDNA viral communities (Fig. 1B). A notable exception was Cellulophaga phage phi38:2, for which relative abundance was systematically higher (2- to 3-fold) in A-LA than TAG samples (Fig. 1B). This genome did not have unusual size or GC content compared to the others (Table

245 1), so the mechanism for this deviation remains unclear.

Second, coverage variation along each genome indicated that MDA coverage was significantly more variable than A-LA and TAG for all genomes but one, and TAG more variable than A-LA for 6 of 10 genomes (Wilcoxon test, p-value $>0.01$, effect $s i z e<0.8$ ), with highly variable coverage in TAG datasets for low GC genomes (Fig. S3, Table S3). Thus, among the tested

250 methods, the A-LA protocol produces the most even coverage across dsDNA viral genomes.

In summary, these mock community findings suggest that A-LA was uniquely able to quantitatively recover ssDNA virus relative abundances from both mock communities, and also more quantitatively represented the coverage within dsDNA genomes. This indicates that A-LA would be the library preparation method of choice when targeting both ssDNA and dsDNA

255 viruses in surveys of natural communities.

Estimating the contribution of ssDNA viruses to aquatic viral communities 
Given promising mock community benchmarking results, we next sought to apply these methods to establish their performances on natural communities, and to obtain first estimates of ssDNA virus sequence abundance in nature. To this end, we generated viromes for three

260 freshwater and three seawater samples using the same library preparation protocols as above (MDA, TAG, A-LA; Supplementary Material \& Methods). Overall, ssDNA viruses were detected in all samples, although these amounted only to 33 - 370 contigs in any given sample as compared to 14,000 - 99,000 dsDNA contigs (Table S4). However, because aquatic viruses are vastly under-represented in databases, a large proportion (35-71\%) of the assembled contigs could not be confidently affiliated to either ssDNA or dsDNA viruses. Hence, we chose to generate a less stringent estimation of ssDNA contigs by adding all contigs not detected in TAG libraries (12,134 to 53,950 contigs, Table S4) to these BLAST-affiliated ssDNA sequences. Our reasoning is that unknown contigs detected in MDA or A-LA libraries (which will include ssDNA templates) and not in TAG libraries (strongly biased against ssDNA templates) likely originate from ssDNA genomes.

Based on A-LA viromes, which mock community experiments suggested were the most quantitative, the relative abundance (estimated through read coverage) of ssDNA contigs (conservatively identified by best BLAST hit to ssDNA virus genomes) was $0.03-4.68 \%$ and $0.005-0.03 \%$ in freshwater and seawater viral communities, respectively (Fig. 2A, Table S5).

275 Meanwhile, the putative new ssDNA viruses (i.e. A-LA/MDA-only unknown contigs) could account for as much as $1.91-68.00 \%$ of freshwater and $12.15-15.98 \%$ of seawater DNA viral communities (Fig. 2A). Because this class of contigs might also include rare dsDNA viruses that would be haphazardly represented and not detected due to chance in the TAG libraries, these values of ssDNA abundance should be treated as lower and upper bounds. In addition, these 280 upper bounds are likely over-estimations, especially in samples where few TAG reads are available, such as SU08M from Lake Superior (total ssDNA fraction estimated at 72.68\%). Nevertheless, these still suggest that ssDNA viruses are less abundant than dsDNA viruses in 4 of 6 aquatic samples tested here (Fig. 2A, Table S5) although further work is required to address the recovery efficiency of ssDNA vs dsDNA viruses using various concentration and DNA

285 extraction methods, as well as compare their decay rate and stability under different storage conditions, since both could influence the relative abundance of ssDNA vs dsDNA viruses in viromes.

Consistent with the mock community experiments, ssDNA viral genomes were systematically over-represented $>10$-fold in MDA viromes and under-represented $>10$-fold in TAG viromes, 290 relative to A-LA (Table S5). This impacts rank-abundance distributions such that identifiable ssDNA viruses rank among the 10 most abundant contigs in MDA viromes, but are much lower ranked $(\sim 1,000-35,000$ th most abundant viruses) in A-LA viromes, and near or below detection limits in TAG viromes (Table S5). The only exception is sample SU08M, where ssDNA viruses rank as high as the 19th most abundant contig and have 16 additional viruses in the 100 most 295 abundant viral sequences in the A-LA virome. These abundant ssDNA viruses included bacteriophages (from the Microviridae family) and eukaryotic circular Rep-encoding ssDNA (CRESS-DNA) viruses (Rosario et al., 2012) (Table S6). Thus, even when ssDNA viruses as a whole do not represent a large part of the DNA viral community (affiliated ssDNA viruses account for only $3.68 \%$ of the reads in this sample), individual ssDNA viruses can be abundant.

300 The MDA bias towards enrichment for ssDNA viruses can be a positive attribute: MDA libraries captured 2-15 times more ssDNA viral genomes ("affiliated" ssDNA) than A-LA in 4 out of 6 samples (Fig. 2B). The two samples where MDA captured fewer ssDNA viruses 
represent unique situations: sample T102S had very few ssDNA viruses in any of its viromes, and the MDA library for sample SU08M was smaller by an order of magnitude relative to A-LA

305 library due to multiplexing issues in the sequencing run (Table S5). Thus, when ssDNA viruses were available in the samples, and where sequencing depth was relatively consistent across library prep methods, MDA remains the clear method of choice to maximally enrich for ssDNA viruses if quantitative comparisons are not needed.

\section{Conclusion}

310 The description of a large unsuspected genetic diversity of ssDNA viruses across multiple ecosystems (Ge et al., 2011; Kim et al., 2011; Rosario, Duffy \& Breitbart, 2012; Labonté \& Suttle, 2013b; Eaglesham \& Hewson, 2013; Quaiser et al., 2015; Dayaram et al., 2016), and unique evolutionary patterns including gene exchanges between RNA and DNA genomes (Krupovic, Ravantti \& Bamford, 2009; Diemer \& Stedman, 2012), have highlighted ssDNA

315 viruses as one of the most intriguing viral groups in viral ecology. So far, two main types of ssDNA viruses have been frequently detected in viromes: eukaryote-infecting CRESS-DNA viruses and bacteriophages from the Microviridae family. Novel and unusual ssDNA viruses continue to be isolated, particularly from eukaryotic and archaeal hosts (Tomaru et al., 2012; Mochizuki et al., 2012; Kimura \& Tomaru, 2015). However, the relative abundance of ssDNA

320 viruses among DNA viral communities remains an open and challenging question to address. Here, the use of A-LA library preparation protocol enabled us to quantify (+/- 1.8 -fold) the fraction of ssDNA and dsDNA viruses in natural communities. This revealed that ssDNA viruses are consistently present, but outnumbered by dsDNA viruses in all 6 aquatic samples tested. Nevertheless, individual ssDNA viruses were occasionally abundant, even when dsDNA viruses

325 dominated the community. Hence, combined with the fact that ssDNA viruses likely infect a broad host range distinct from those of dsDNA viruses, the former should not be overlooked when investigating whole environmental viral communities and their impact on ecosystems. 
Figures

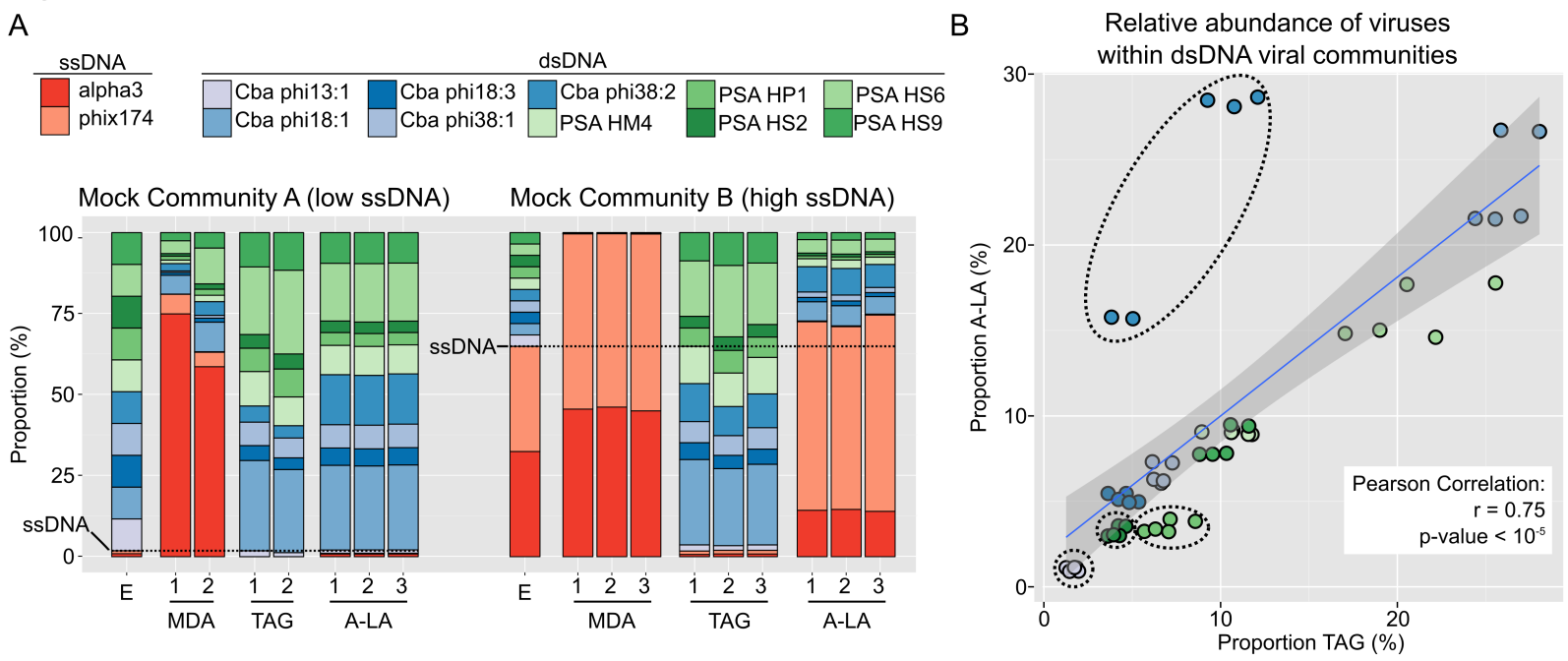

Figure 1: Comparison of amplification efficiency for ssDNA and dsDNA genomes of Multiple Displacement Amplification (MDA), Tagmentation (TAG) and Adaptase-Linker Amplification (A-LA) from mock community samples. A. Composition of mock communities' viromes prepared with MDA, TAG, and A-LA. For each community, the first bar displays the expected proportion of each virus ("E"), and replicates are noted with a number (1-3) when available. Expected proportions of ssDNA viruses are highlighted with a dashed horizontal line

$335(1.8 \%$ and $64.9 \%$ of $\mathrm{MCA}$ and $\mathrm{MCB}$ respectively). B. Correlation between the relative abundance of individual dsDNA viruses (within the dsDNA viral community) in TAG (x-axis) and A-LA (y-axis) viromes. The color code of circles is the same as in Fig. 1A. Genomes for which the relative abundance distributions are significantly different in TAG vs A-LA are highlighted with dotted outline (Fig. S2).
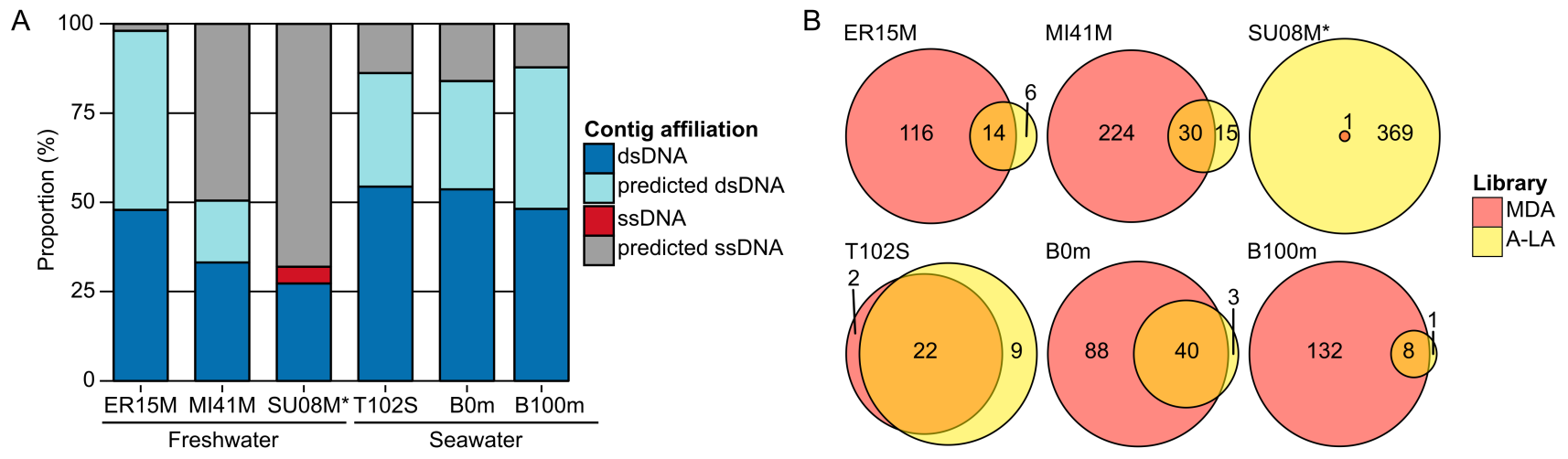

340 Figure 2: Relative abundance of ssDNA vs dsDNA viruses in freshwater and seawater samples and estimated diversity of ssDNA viruses. A. Composition of A-LA viromes from 6 aquatic samples (based on the read coverage of assembled contigs). Contigs were affiliated based on best BLAST hit to NCBI RefSeq Virus ("dsDNA" and "ssDNA" contigs) or if not possible (no significant hit or mixed hits to both dsDNA and ssDNA genomes), based on their coverage in 
345 the TAG virome ("predicted dsDNA" if covered in TAG virome, "predicted ssDNA" otherwise). Relative abundance was calculated based on the coverage of each contig by virome reads. ER15M: Lake Erie, MI41M: Lake Michigan, SU08M: Lake Superior, T102S: surface sample of station Tara Ocean 102, B0m and B100m: surface and 100m-deep samples from the Bermuda Atlantic Time-series Study site collected in March 2011. B. Comparison of ssDNA viruses 350 contigs recovered in MDA and A-LA library. For each sample, a Venn diagram depicts the number of contigs affiliated to ssDNA detected in MDA, A-LA, and both libraries (contigs detection based on a mapping of the library reads). *For sample SU08M, a limited number of quality-controlled reads were available for MDA and LA libraries $(\sim 1$ order of magnitude less than for other samples).

\begin{tabular}{ccccccccc}
\hline Genome type & Phage & Family & Host & $\begin{array}{c}\text { Genome } \\
\text { length (bp) }\end{array}$ & GC\% & $\begin{array}{c}\text { Theoretical } \\
\text { proportion in } \\
\text { MCA (low } \\
\text { ssDNA) }\end{array}$ & $\begin{array}{c}\text { Theoretical } \\
\text { proportion in } \\
\text { MCB (high } \\
\text { ssDNA) }\end{array}$ & $\begin{array}{c}\text { NCBI } \\
\text { genome Id }\end{array}$ \\
\hline dsDNA & PSA-HM4 & Myoviridae & PSA & 129396 & $35.71 \%$ & $9.82 \%$ & $3.51 \%$ & KF302034 \\
dsDNA & PSA-HP1 & Podoviridae & PSA & 45035 & $44.69 \%$ & $9.82 \%$ & $3.51 \%$ & KF302037 \\
dsDNA & PSA-HS9 & Siphoviridae & PSA & 36769 & $40.50 \%$ & $9.82 \%$ & $3.51 \%$ & KF302033 \\
dsDNA & PSA-HS2 & Siphoviridae & PSA & 37728 & $40.21 \%$ & $9.82 \%$ & $3.51 \%$ & KF302036 \\
dsDNA & PSA-HS6 & Siphoviridae & PSA & 35328 & $44.78 \%$ & $9.82 \%$ & $3.51 \%$ & KF302035 \\
dsDNA & Cba phi38:1 & Podoviridae & Cba & 72534 & $38.05 \%$ & $9.82 \%$ & $3.51 \%$ & NC_021796 \\
dsDNA & Cba phi18:3 & Podoviridae & Cba & 71443 & $32.86 \%$ & $9.82 \%$ & $3.51 \%$ & NC_021794 \\
dsDNA & Cba phi38:2 & Myoviridae & Cba & 54012 & $33.17 \%$ & $9.82 \%$ & $3.51 \%$ & KC821629 \\
dsDNA & Cba phi13:1 & Siphoviridae & Cba & 76666 & $30.23 \%$ & $9.82 \%$ & $3.51 \%$ & KC821625 \\
dsDNA & Cba phi18:1 & Siphoviridae & Cba & 39189 & $36.29 \%$ & $9.82 \%$ & $3.51 \%$ & NC_021790 \\
ssDNA & phix174 & Microviridae & E. coli & 5386 & $44.80 \%$ & $0.92 \%$ & $32.47 \%$ & NC_001422 \\
ssDNA & alpha3 & Microviridae & E. coli & 6089 & $44.56 \%$ & $0.92 \%$ & $32.47 \%$ & NC_001330 \\
\hline
\end{tabular}

355 Table 1: Characteristics of phage genomes included in the mock communities. PSA: Pseudoalteromonas, Cba: Cellulophaga baltica. E. coli: Escherichia coli. 


\section{Supplementary Figures}

Figure S1: Transmission electron micrographs of Microviridae alpha3 (A) and phiX174 (B) stained with SYBR Gold. Examples of spots counted as individual viruses are indicated with an arrow (5 for each panel).

Figure S2: Variability of average coverage of dsDNA viruses in mock community viromes. The relative abundance of each dsDNA viral genome within the dsDNA viral community (i.e. excluding read mapped to ssDNA viruses) was estimated for each virus in each sample. These relative abundance values are plotted for grouped by sample (left panel), or grouped by genome

365 (right panel). Sample methods (MDA, TAG or A-LA) are indicated via coloring of the boxplot (left panel) or dot (right panel). Genomes with relative abundance significantly different between TAG and A-LA libraries (Wilcoxon two-sided test) are indicated with a star above the boxplot (right panel).

Figure S3: Variation of coverage (y-axis) along individual dsDNA genomes (x-axis). The coverage was estimated for each genome on sliding windows of $1,000 \mathrm{bp}$ separated by a $100 \mathrm{bp}$ step. The variability of coverage was then estimated for each sample and each genome by calculating the coverage coefficient of variation (standard deviation divided by average). On the $\mathrm{x}$-axis, genomes are ordered according to their overall GC content. All pairs of distributions are significantly different (Wilcoxon two-sided test) except for the ones indicated as "ns".

\section{Supplementary Tables}

Table S1: DNA extraction efficiency for dsDNA phages, and comparison between SYBR counts and PFU estimations of the VLP concentration for ssDNA phages

Table S2: Relative abundance and coverage of phage genomes in libraries from mock communities. The relative abundance of each genome was calculated based on its coverage, taking into account that each copy of dsDNA genome would produce twice as much coverage as a copy of ssDNA genome (because of the initial denaturation step). For comparing the efficiency of different methods for each group of phage (i.e. dsDNA and ssDNA), relative abundance within each group were computed from this table (by dividing the value for each phage by the sum for the group). The raw coverage for each genome in each sample is indicated in the bottom 385 table.

Table S3: Accuracy and efficiency of mock community genome coverage and assembly in ALA viromes. The coverage of each genome across the different contigs is indicated for each library (\% covered) alongside the coefficient of variation in read-based coverage along the genomes (Coverage CV) and the number of contigs matching this genome (\# contigs). A star $\left(^{*}\right)$ denotes libraries for which the assembly had to be run on a subset of the QC'd reads (random subsamples of 1,000,000 reads) to avoid biases linked to the over-coverage of ssDNA genomes.

Table S4: Cross-assembly result of freshwater and seawater viromes. For each sample, the number of contigs assembled ( $\geq 500 \mathrm{bp}$ ) classified into ssDNA contigs, dsDNA contigs, predicted 
ssDNA contigs, and predicted dsDNA contigs is indicated. Predicted ssDNA or dsDNA contigs are sequences for which a gene-base affiliation was not possible (either because the predicted genes did not have any similarity to a known viral genome, or because these included similarities to both ssDNA and dsDNA genomes) and which genome type were defined based on the coverage across libraries: the sequence was considered dsDNA if covered in TAG library, ssDNA otherwise (i.e. only covered in MDA and/or A-LA). Sample codes are the same as in Fig. 2.

400 Table S5: Relative abundance of the four different types of contigs (ssDNA, dsDNA, predicted ssDNA and predicted dsDNA) in each virome. Relative abundances are based on normalized coverage of the contigs (i.e. average coverage across the contig divided by the total number of bp sequenced in the library). Sample codes are the same as in Fig. 2.

Table S6: Affiliation of the most abundant affiliated sSDNA virus contigs in sample SU08M

405 (A-LA library). Contigs were ranked based on their read coverage in the library. Affiliations are based on best BLAST hit against NCBI RefSeqVirus, and presented for the 17 contigs affiliated to ssDNA viruses within the 100 most abundant contigs. CRESS-DNA: circular Rep-encoding single-stranded DNA.

\section{Acknowledgements}

410 Seawater samples were available thanks to the Tara Oceans expedition and BATS cruises. We thank Dr. Bentley Fane from University of Arizona for providing phiX174 and alpha3 strains. We acknowledge support from the Ohio Supercomputer Center.

\section{References}

Aigrain L, Gu Y, Quail M a. 2016. Quantitation of next generation sequencing library preparation protocol efficiencies using droplet digital PCR assays - a systematic comparison of DNA library preparation kits for Illumina sequencing. BMC genomics 17:458. DOI: 10.1186/s12864-016-2757-4.

Angly FE, Felts B, Breitbart M, Salamon P, Edwards RA, Carlson C, Chan AM, Haynes M, Kelley S, Liu H, Mahaffy JM, Mueller JE, Nulton J, Olson R, Parsons R, Rayhawk S, Suttle CA, Rohwer F. 2006. The marine viromes of four oceanic regions. PLoS biology 4:e368.

Angly FE, Willner D, Prieto-Davó A, Edwards RA, Schmieder R, Vega-Thurber R, Antonopoulos DA, Barott K, Cottrell MT, Desnues C, Dinsdale EA, Furlan M, Haynes M, Henn MR, Hu Y, Kirchman DL, McDole T, McPherson JD, Meyer F, Miller RM, Mundt E, Naviaux RK, Rodriguez-Mueller B, Stevens R, Wegley L, Zhang L, Zhu B, Rohwer F. 2009. The GAAS metagenomic tool and its estimations of viral and microbial average genome size in four major biomes. PLoS computational biology 5:e1000593. DOI: 10.1371/journal.pcbi.1000593.

Bankevich A, Nurk S, Antipov D, Gurevich A a, Dvorkin M, Kulikov AS, Lesin VM, Nikolenko SI, Pham S, Prjibelski AD, Pyshkin A V, Sirotkin A V, Vyahhi N, Tesler G, Alekseyev M a, 
molecular cell biology 19:455-77. DOI: 10.1089/cmb.2012.0021.

Bolger AM, Lohse M, Usadel B. 2014. Trimmomatic: a flexible trimmer for Illumina sequence data. Bioinformatics (Oxford, England) 30:2114-2120. DOI: 10.1093/bioinformatics/btu170.

Brown CT, Hug L a., Thomas BC, Sharon I, Castelle CJ, Singh A, Wilkins MJ, Wrighton KC, Williams KH, Banfield JF. 2015. Unusual biology across a group comprising more than 15\% of domain Bacteria. Nature 523:208-211. DOI: 10.1038/nature14486.

Brum J, Ignacio-Espinoza J, Roux S, Doulcier G, Acinas SG, Alberti A, Chaffron S, Cruaud C, de Vargas C, Gasol JM, Gorsky G, Gregory AC, Ogata H, Pesant S, Poulos BT, Schwenck SM, Speich S, Dimier C, Kandels-Lewis S, Picheral M, Searson S, Coordinators TO, Bork P, Bowler C, Sunagawa S, Wincker P, Karsenti E, Sullivan MB. 2015. Patterns and ecological drivers of ocean viral communities. Science 348:1261498-1-10. DOI: 10.1126/science. 1261498 .

Brum JR, Sullivan MB. 2015. Rising to the challenge: accelerated pace of discovery transforms marine virology. Nature Reviews Microbiology 13:147-59. DOI: 10.1038/nrmicro3404.

Castelle CJ, Hug LA, Wrighton KC, Thomas BC, Williams KH, Wu D, Tringe SG, Singer SW, Eisen JA, Banfield JF. 2013. Extraordinary phylogenetic diversity and metabolic versatility in aquifer sediment. Nature communications 4:2120. DOI: 10.1038/ncomms3120.

Cunningham BR, Brum JR, Schwenck SM, Sullivan MB, John SG. 2015. An inexpensive, accurate, and precise wet-mount method for enumerating aquatic viruses. Applied and environmental microbiology 81:2995-3000. DOI: 10.1128/AEM.03642-14.

Dayaram A, Galatowitsch ML, Argüello-Astorga GR, van Bysterveldt K, Kraberger S, Stainton D, Harding JS, Roumagnac P, Martin DP, Lefeuvre P, Varsani A. 2016. Diverse circular replication-associated protein encoding viruses circulating in invertebrates within a lake ecosystem. Infection, genetics and evolution : journal of molecular epidemiology and evolutionary genetics in infectious diseases 39:304-16. DOI: 10.1016/j.meegid.2016.02.011.

Delcher AL, Salzberg SL, Phillippy AM. 2003. Using MUMmer to identify similar regions in large sequence sets. Current protocols in bioinformatics 00:10.3:10.3.1-10.3.18. DOI: 10.1002/0471250953.bi1003s00.

Diemer GS, Stedman KM. 2012. A novel virus genome discovered in an extreme environment suggests recombination between unrelated groups of RNA and DNA viruses. Biology direct 7:13. DOI: $10.1186 / 1745-6150-7-13$. genome landscapes of marine Pseudoalteromonas phage H105/1. The ISME journal 5:107- 
21. DOI: $10.1038 /$ ismej.2010.94.

Duhaime MB, Deng L, Poulos BT, Sullivan MB. 2012. Towards quantitative metagenomics of wild viruses and other ultra-low concentration DNA samples: a rigorous assessment and optimization of the linker amplification method. Environmental microbiology 14:2526-37. DOI: $10.1111 / j .1462-2920.2012 .02791 . x$.

Eaglesham J, Hewson I. 2013. Widespread detection of circular replication initiator protein (rep)encoding ssDNA viral genomes in estuarine, coastal and open ocean net plankton. Marine Ecology Progress Series 494:65-72. DOI: 10.3354/meps10575.

475 Edwards RA, Rohwer F. 2005. Viral metagenomics. Nature Reviews Microbiology 3:504-510.

Falkowski PG, Fenchel T, Delong EF. 2008. The Microbial Engines That Drive Earth's Biogeochemical Cycles. Science 320:1034-9. DOI: 10.1126/science.1153213.

Fancello L, Raoult D, Desnues C. 2012. Computational tools for viral metagenomics and their application in clinical research. Virology 434:162-174. DOI: 10.1016/j.virol.2012.09.025.

Fuhrman JA. 1999. Marine viruses and their biogeochemical and ecological effects. Nature 399:541-548. DOI: 10.1038/21119.

Ge X, Li J, Peng C, Wu L, Yang X, Wu Y, Zhang Y, Shi Z. 2011. Genetic diversity of novel circular ssDNA viruses in bats in China. The Journal of general virology 92:2646-53. DOI: 10.1099/vir.0.034108-0.

485 Goldsmith D, Brum J, Hopkins M, Carlson C, Breitbart M. 2015. Water column stratification structures viral community composition in the Sargasso Sea. Aquatic Microbial Ecology 76:85-94. DOI: 10.3354/ame01768.

Holmfeldt K, Odić D, Sullivan MB, Middelboe M, Riemann L. 2012. Cultivated single-stranded DNA phages that infect marine Bacteroidetes prove difficult to detect with DNA-binding stains. Applied and environmental microbiology 78:892-4. DOI: 10.1128/AEM.06580-11.

Holmfeldt K, Solonenko N, Shah M, Corrier K, Riemann L, VerBerkmoes NC, Sullivan MB. 2013. Twelve previously unknown phage genera are ubiquitous in the global oceans. Proceedings of the National Academy of Sciences of the United States of America 110:12798-12803. DOI: 10.1073/pnas.1305956110.

495 Hug L a., Baker BJ, Anantharaman K, Brown CT, Probst AJ, Castelle CJ, Butterfield CN, Hernsdorf AW, Amano Y, Ise K, Suzuki Y, Dudek N, Relman D a., Finstad KM, Amundson R, Thomas BC, Banfield JF. 2016. A new view of the tree of life. Nature Microbiology:16048. DOI: 10.1038/nmicrobiol.2016.48.

John SG, Mendez CB, Deng L, Poulos B, Kauffman AKM, Kern S, Brum J, Polz MF, Boyle E a, 500 Sullivan MB. 2011. A simple and efficient method for concentration of ocean viruses by 
chemical flocculation. Environmental microbiology reports 3:195-202. DOI: 10.1111/j.1758-2229.2010.00208.x.

Kim K-H, Chang H-W, Nam Y-D, Roh SW, Kim M-S, Sung Y, Jeon CO, Oh H-M, Bae J-W. 2008. Amplification of uncultured single-stranded DNA viruses from rice paddy soil. Applied and environmental microbiology 74:5975-5985. DOI: 10.1128/AEM.01275-08.

Kim M-S, Park E-J, Roh SW, Bae J-W. 2011. Diversity and abundance of single-stranded DNA viruses in human feces. Applied and environmental microbiology 77:8062-70. DOI: 10.1128/AEM.06331-11.

Kim K-H, Bae J-W. 2011. Amplification methods bias metagenomic libraries of uncultured single-stranded and double-stranded DNA viruses. Applied and environmental microbiology 77:7663-8. DOI: 10.1128/AEM.00289-11.

Kimura K, Tomaru Y. 2015. Discovery of two novel viruses expands the diversity of singlestranded DNA and single-stranded RNA viruses infecting a cosmopolitan marine diatom. Applied and environmental microbiology 81:1120-31. DOI: 10.1128/AEM.02380-14.

515 Krupovic M, Ravantti JJ, Bamford DH. 2009. Geminiviruses: a tale of a plasmid becoming a virus. BMC evolutionary biology 9:112. DOI: 10.1186/1471-2148-9-112.

Kurihara L, Banks L, Chupreta S, Couture C, Laliberte J, Sandhu S, Schumacher C, Spurbeck R, Makarov V. 2014. A new method for low-input, PCR-free NGS libraries. Available at http://www.swiftbiosci.com/_literature_170185/A_new_method_for_low-input,_PCRfree NGS libraries (accessed 21 September 2016).

Labonté JM, Suttle C a. 2013a. Metagenomic and whole-genome analysis reveals new lineages of gokushoviruses and biogeographic separation in the sea. Frontiers in Microbiology 4:404. DOI: 10.3389/fmicb.2013.00404.

Labonté JM, Suttle CA. 2013b. Previously unknown and highly divergent ssDNA viruses 525 populate the oceans. The ISME journal 7:2169-77. DOI: 10.1038/ismej.2013.110.

Langmead B, Salzberg SL. 2012. Fast gapped-read alignment with Bowtie 2. Nature methods 9:357-9. DOI: 10.1038/nmeth.1923.

López-Bueno A, Tamames J, Velázquez D, Moya A, Quesada A, Alcamí A. 2009. High Diversity of the Viral Community from an Antarctic Lake. Science 326:858-861. DOI: 10.1126/science.1179287.

Male MF, Kraberger S, Stainton D, Kami V, Varsani A. 2016. Cycloviruses, gemycircularviruses and other novel replication-associated protein encoding circular viruses in Pacific flying fox (Pteropus tonganus) faeces. Infection, genetics and evolution : journal of molecular epidemiology and evolutionary genetics in infectious diseases 39:279-92. DOI: 
Marine R, McCarren C, Vorrasane V, Nasko D, Crowgey E, Polson SW, Wommack KE. 2014. Caught in the middle with multiple displacement amplification: the myth of pooling for avoiding multiple displacement amplification bias in a metagenome. Microbiome 2:3. DOI: 10.1186/2049-2618-2-3.

540 Mochizuki T, Krupovic M, Pehau-Arnaudet G, Sako Y, Forterre P, Prangishvili D. 2012. Archaeal virus with exceptional virion architecture and the largest single-stranded DNA genome. Proceedings of the National Academy of Sciences of the United States of America 109:1-6. DOI: 10.1073/pnas.1203668109.

Mokili JL, Rohwer F, Dutilh BE. 2012. Metagenomics and future perspectives in virus discovery. Current Opinion in Virology 2:63-77. DOI: 10.1016/j.coviro.2011.12.004.

Ng TFF, Manire C, Borrowman K, Langer T, Ehrhart L, Breitbart M. 2009. Discovery of a novel single-stranded DNA virus from a sea turtle fibropapilloma by using viral metagenomics. Journal of virology 83:2500-9. DOI: 10.1128/JVI.01946-08.

Noble RT. 2001. Enumeration of viruses. Methods in Microbiology 30:43-51. DOI: dx.doi.org/10.1016/S0580-9517(01)30038-7.

O’Malley M a. 2016. The ecological virus. Studies in history and philosophy of biological and biomedical sciences:1-9. DOI: 10.1016/j.shpsc.2016.02.012.

Pace NR. 1997. A Molecular View of Microbial Diversity and the Biosphere. Science 276:73440. DOI: $10.1126 /$ science.276.5313.734.

555 Pesant S, Not F, Picheral M, Kandels-Lewis S, Le Bescot N, Gorsky G, Iudicone D, Karsenti E, Speich S, Troublé R, Dimier C, Searson S, Acinas SG, Bork P, Boss E, Bowler C, De Vargas C, Follows M, Grimsley N, Hingamp P, Jaillon O, Karp-Boss L, Krzic U, Ogata H, Raes J, Reynaud EG, Sardet C, Sieracki M, Stemmann L, Sullivan MB, Sunagawa S, Velayoudon D, Weissenbach J, Wincker P. 2015. Open science resources for the discovery and analysis of Tara Oceans data. Scientific Data 2:150023. DOI: 10.1038/sdata.2015.23.

Quaiser A, Dufresne A, Ballaud F, Roux S, Zivanovic Y, Colombet J, Sime-Ngando T, Francez A-J. 2015. Diversity and comparative genomics of Microviridae in Sphagnum- dominated peatlands. Frontiers in microbiology 6:375. DOI: 10.3389/fmicb.2015.00375.

R Core Team. 2016. R: A Language and Environment for Statistical Computing. Vienna, Austria: $\mathrm{R}$ Foundation for Statistical Computing.

Rinke C, Schwientek P, Sczyrba A, Ivanova NN, Anderson IJ, Cheng J-F, Darling A, Malfatti S, Swan BK, Gies E a, Dodsworth J a, Hedlund BP, Tsiamis G, Sievert SM, Liu W-T, Eisen J a, Hallam SJ, Kyrpides NC, Stepanauskas R, Rubin EM, Hugenholtz P, Woyke T. 2013. 
Insights into the phylogeny and coding potential of microbial dark matter. Nature 499:4317. DOI: 10.1038 /nature12352.

Rohwer F, Thurber RV. 2009. Viruses manipulate the marine environment. Nature 459:207-212.

Rokyta DR, Burch CL, Caudle SB, Wichman HA. 2006. Horizontal gene transfer and the evolution of microvirid coliphage genomes. Journal of bacteriology 188:1134-1142.

Rosario K, Nilsson C, Lim YW, Ruan Y, Breitbart M. 2009. Metagenomic analysis of viruses in reclaimed water. Environmental microbiology 11:2806-20. DOI: 10.1111/j.14622920.2009.01964.x.

Rosario K, Dayaram A, Marinov M, Ware J, Kraberger S, Stainton D, Breitbart M, Varsani A. 2012. Diverse circular ssDNA viruses discovered in dragonflies (Odonata: Epiprocta). The Journal of general virology 93:2668-81. DOI: 10.1099/vir.0.045948-0.

580 Rosario K, Duffy S, Breitbart M. 2009. Diverse circovirus-like genome architectures revealed by environmental metagenomics. Journal of general virology 90:2418-2424.

Rosario K, Duffy S, Breitbart M. 2012. A field guide to eukaryotic circular single-stranded DNA viruses: insights gained from metagenomics. Archives of virology 157:1851-71. DOI: 10.1007/s00705-012-1391-y.

585 Roux S, Enault F, Robin A, Ravet V, Personnic S, Theil S, Colombet J, Sime-Ngando T, Debroas D. 2012a. Assessing the Diversity and Specificity of Two Freshwater Viral Communities through Metagenomics. PLoS One 7:e33641.

Roux S, Krupovic M, Poulet A, Debroas D, Enault F. 2012b. Evolution and diversity of the Microviridae viral family through a collection of 81 new complete genomes assembled from virome reads. PLoS One 7:e40418. DOI: 10.1371/journal.pone.0040418.

Roux S, Enault F, Hurwitz BL, Sullivan MB. 2015. VirSorter: mining viral signal from microbial genomic data. PeerJ 3:e985. DOI: 10.7717/peerj.985.

Sommer F, Bäckhed F. 2013. The gut microbiota--masters of host development and physiology. Nature reviews. Microbiology 11:227-38. DOI: 10.1038/nrmicro2974.

595 Steel O, Kraberger S, Sikorski A, Young LM, Catchpole RJ, Stevens AJ, Ladley JJ, Coray DS, Stainton D, Dayaram A, Julian L, van Bysterveldt K, Varsani A. 2016. Circular replicationassociated protein encoding DNA viruses identified in the faecal matter of various animals in New Zealand. Infection, genetics and evolution : journal of molecular epidemiology and evolutionary genetics in infectious diseases 43:151-164. DOI:

$600 \quad$ 10.1016/j.meegid.2016.05.008.

Suttle CA. 2007. Marine viruses--major players in the global ecosystem. Nature Reviews Microbiology 5:801-812. 
Tomaru Y, Toyoda K, Kimura K, Hata N, Yoshida M, Nagasaki K. 2012. First evidence for the existence of pennate diatom viruses. The ISME journal 6:1445-8. DOI:

605 10.1038/ismej.2011.207.

Wegley L, Edwards R, Rodriguez-Brito B, Liu H, Rohwer F. 2007. Metagenomic analysis of the microbial community associated with the coral Porites astreoides. Environmental microbiology 9:2707-19. DOI: 10.1111/j.1462-2920.2007.01383.x.

Weitz JS, Stock C a, Wilhelm SW, Bourouiba L, Coleman ML, Buchan A, Follows MJ, Fuhrman 610 J a, Jover LF, Lennon JT, Middelboe M, Sonderegger DL, Suttle C a, Taylor BP, Frede Thingstad T, Wilson WH, Eric Wommack K. 2015. A multitrophic model to quantify the effects of marine viruses on microbial food webs and ecosystem processes. The ISME Journal 9:1352-1364. DOI: 10.1038/ismej.2014.220.

Wickham H. 2009. ggplot2: Elegant Graphics for Data Analysis. Springer Publishing Company.

615 Willner D, Furlan M, Haynes M, Schmieder R, Angly FE, Silva J, Tammadoni S, Nosrat B, Conrad D, Rohwer F. 2009. Metagenomic analysis of respiratory tract DNA viral communities in cystic fibrosis and non-cystic fibrosis individuals. PLoS One 4:e7370.

Wrighton K, Thomas B, Sharon I, Miller CS, Castelle CJ, VerBerkmoes NC, Wilkins MJ, Hettich RL, Lipton MS, Williams KH, Long PE, Banfield JF. 2012. Fermentation, hydrogen, and sulfur metabolism in multiple uncultivated bacterial phyla. Science 337:1661-1666.

Yilmaz S, Allgaier M, Hugenholtz P. 2010. Multiple displacement amplification compromises quantitative analysis of metagenomes. Nature methods 7:943-4. DOI: 10.1038/nmeth1210943.

Zawar-Reza P, Argüello-Astorga GR, Kraberger S, Julian L, Stainton D, Broady P a, Varsani A. 625 2014. Diverse small circular single-stranded DNA viruses identified in a freshwater pond on the McMurdo Ice Shelf (Antarctica). Infection, genetics and evolution : journal of molecular epidemiology and evolutionary genetics in infectious diseases 26:132-8. DOI: 10.1016/j.meegid.2014.05.018. 